\title{
Erratum: Dynamic Stratification in Drying Films of Colloidal Mixtures [Phys. Rev. Lett. 116, 118301 (2016)]
}

\begin{abstract}
Andrea Fortini, Ignacio Martín-Fabiani, Jennifer Lesage De La Haye, Pierre-Yves Dugas, Muriel Lansalot, Franck D’Agosto, Elodie Bourgeat-Lami, Joseph L. Keddie, and Richard P. Sear

(Received 9 May 2016; published 2 June 2016)
\end{abstract}

DOI: 10.1103/PhysRevLett.116.229901

After publication, some inaccuracies and typos were found.

In Fig. 4(b), the gradient of osmotic pressure is rescaled by the parameter $\beta$, which is not defined in the Letter. The parameter $\beta=1 / k_{B} T$, where $k_{B}$ is the Boltzmann constant and $T$ is the temperature.

There are two typographical errors in Eq. (5) of the Supplemental Material. The correct equation for the potential used in the simulations is

$$
\frac{U_{i}(z)}{k_{B} T}= \begin{cases}\frac{\alpha_{i}}{k_{B} T}\left(z-\left[z_{\mathrm{int}}(t)-r_{0}\right]\right)^{2}, & z>z_{\text {int }}(t)-r_{0} \\ 0, & \text { otherwise. }\end{cases}
$$

The strength of the air-water interface attraction reported in the Letter was not correctly scaled with the temperature. The value used in the simulations is $\alpha_{i} / k_{B} T=25\left(d_{i} / d_{s}\right)^{2}$. Likewise, the contact value of the particle-wall interaction $U_{i w}(z)$ is $\epsilon_{w} / k_{B} T=2.5$. The correct contact value of the particle-particle interaction used in the simulations is $\epsilon / k_{B} T=0.625$.

The inaccuracies in the Supplemental Material were brought to our attention by Athanassios Z. Panagiotopoulos, Arash Nikoubashman, and Mike Howard. 